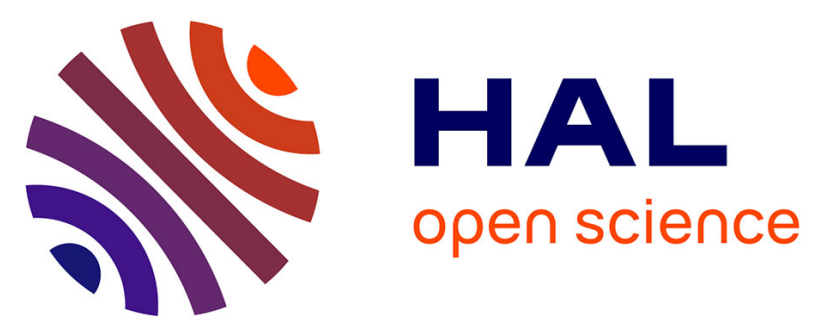

\title{
Polyglutamic acid-PEG nanocapsules as long circulating carriers for the delivery of docetaxel
}

Giovanna Lollo, Gustavo Rivera-Rodriguez, Jérôme Bejaud, Tristan Montier,

Catherine Passirani-Malleret, Jean-Pierre Benoit, Marcos García-Fuentes, Maria José Alonso, Dolores Torres

\section{To cite this version:}

Giovanna Lollo, Gustavo Rivera-Rodriguez, Jérôme Bejaud, Tristan Montier, Catherine PassiraniMalleret, et al.. Polyglutamic acid-PEG nanocapsules as long circulating carriers for the delivery of docetaxel. European Journal of Pharmaceutics and Biopharmaceutics, 2014, 87 (1), Non spécifié. 10.1016/j.ejpb.2014.02.004 . hal-03179499

\section{HAL Id: hal-03179499 \\ https://univ-angers.hal.science/hal-03179499}

Submitted on 24 Mar 2021

HAL is a multi-disciplinary open access archive for the deposit and dissemination of scientific research documents, whether they are published or not. The documents may come from teaching and research institutions in France or abroad, or from public or private research centers.
L'archive ouverte pluridisciplinaire HAL, est destinée au dépôt et à la diffusion de documents scientifiques de niveau recherche, publiés ou non, émanant des établissements d'enseignement et de recherche français ou étrangers, des laboratoires publics ou privés. 
Research paper

\title{
Polyglutamic acid-PEG nanocapsules as long circulating carriers for the delivery of docetaxel
}

\author{
Giovanna Lollo a,b,1, Gustavo R. Rivera-Rodriguez ${ }^{\mathrm{a}, \mathrm{b}, 2}$, Jerome Bejaud ${ }^{\mathrm{c}}$, Tristan Montier ${ }^{\mathrm{d}, \mathrm{e}}$, \\ Catherine Passirani ${ }^{c}$, Jean-Pierre Benoit ${ }^{c}$, Marcos García-Fuentes ${ }^{a, b}$, Maria José Alonso a,b, \\ Dolores Torres ${ }^{\mathrm{a}, \mathrm{b}, *}$

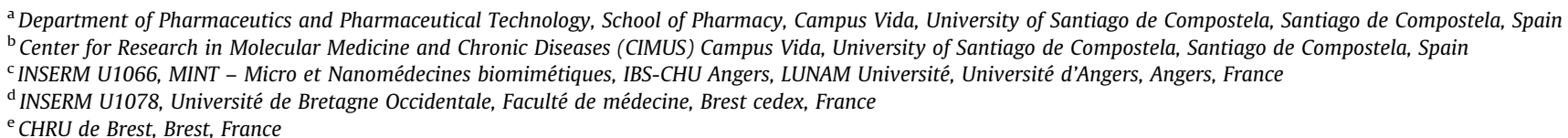

\section{A R T I C L E I N F O}

Article history:

Received 17 August 2013

Accepted in revised form 4 February 2014

Available online $\mathrm{xxxx}$

\section{Keywords:}

Anticancer drug delivery

Biocompatibility

Drug targeting

Nanotechnology

Polyaminoacids

PEGylation

\begin{abstract}
A B S T R A C T
Recently we reported for the first time a new type of nanocapsules consisting of an oily core and a polymer shell made of a polyglutamic acid-polyethylene glycol (PEG-PGA) grafted copolymer with a $24 \% \mathrm{w} / \mathrm{w}$ PEG content. The goal of the work presented here has been to develop a new version of these nanocapsules, in which the shell is made of a di-block PEG-PGA copolymer with a 57\% w/w PEG content and to evaluate their potential for improving the biodistribution and pharmacokinetics of the anticancer drug docetaxel (DCX). A comparative analysis of the biodistribution of fluorescently labeled PGA-PEG nanocapsules versus PGA nanocapsules or a control nanoemulsion (containing the same oil than the nanocapsules) showed that the nanocapsules, and in particular PEGylated nanocapsules, have significantly higher half-life, MRT (Mean Residence Time) and AUC (Area under the Curve) than the nanoemulsion. On a separate set of experiments, PGA-PEG nanocapsules were loaded with DCX and their antitumor efficacy was evaluated in a xenograft U87MG glioma mouse model. The results showed that the survival rate for mice treated with DCX-loaded nanocapsules was significantly increased over the control Taxotere ${ }^{\circledR}$, while the antitumoral effect of both formulations was comparable (60\% tumor growth inhibition with respect to the untreated mice). These results highlight the potential use of these novel nanocapsules as a new drug delivery platform in cancer therapy.
\end{abstract}

(c) 2014 Published by Elsevier B.V.

\section{Introduction}

The number of oncological nanomedicines approved so far has made clear the potential of nanocarriers as a strategy to overcome important drawbacks associated with conventional formulations of anticancer agents [1]. These drawbacks refer mainly to their low

\footnotetext{
* Corresponding author. Department of Pharmaceutics and Pharmaceutical Technology, School of Pharmacy, Campus Vida, University of Santiago de Compostela, 15782 Santiago de Compostela, Spain. Tel.: +34 881814880; fax: +34 981547 148.

E-mail address: dolores.torres@usc.es (D. Torres).

1 Present address: INSERM U1066, MINT - Micro et Nanomédecines biomimétiques, IBS-CHU Angers, LUNAM Université, Université d'Angers, f-49100 Angers, France.

2 Present address: Molecular Phytopathology and Renewable Resources Group, Institut für Biologie und Biotechnologie der Pflanzen, Westfälische WilhelmsUniversität Münster, Schlossplatz 8, 48143 Münster, Germany.
}

water solubility and overwhelming toxicity associated with the lack of selectivity for cancer cells. Often, this toxicity problem has been further enhanced by the use of excipients and solvents, which are themselves responsible for additional serious side effects. Drug delivery nanostructures offer suitable means to improve current cancer chemotherapy by solving these water solubility problems and also by modulating the pharmacokinetics and biodistribution of cytotoxic drugs. Namely, they have the possibility to passively extravasate the fenestrated vasculature of tumor tissues and accumulate in cancer tumor cells. However, this accumulation is greatly dependent on the so-called "stealth" properties of nanocarriers and, thus, on their ability to circulate in the blood stream for prolonged periods of time. These properties have been classically assigned to a variety of nanocarriers, including liposomes [2], PLGA-PEG nanoparticles [3], and polymer micelles [4], by the use of PEGylated biomaterials (either polymers or phospholipids) [5]. However, such protective behavior has been 
recently ascribed to other polymers, i.e. polyaminoacids [6,7]. In addition to their hydrosolubility, polyaminoacids - among them poly-L-glutamic acid (PGA) - are gaining attention because of their biodegradability [8], and overall good safety profile [9,10].

Interestingly, besides their shielding properties, PGA and PEGPGA themselves have been proposed as anti-cancer drug delivery vehicles when presented in the form of micelles or polymer conjugates $[9,10]$. The success of these formulations is exemplified by the fact that two formulations, PGA conjugates and PGA-PEG micelles, are under clinical development $\left(\right.$ Xyotax $^{\circledR} /$ Opaxio $^{\circledR}$ and NC-6004) [11-13]. In the case of micelles, the PEGylation of PGA has been reported to positively influence its inherent shielding properties [14]. This background information has recently encouraged us to design novel delivery carriers based on PGA-PEG known as nanocapsules. These nanocapsules, consisting of an oily core and a PGA-PEG corona, were expected to have specific advantages. Namely, the oily core was intended to allocate significant amounts of hydrophobic drugs and sustain their release, whereas the polymer corona was designed to act as a shield. As predicted, these nanocapsules showed the capacity to encapsulate the hydrophobic anticancer drug plitidepsin. They also improved its pharmacokinetics and toxicity profile over those of the drug in solution [15].

Taking all this into consideration, the purpose of our work has been to develop a type of PGA-PEG nanocapsules, with a different kind of corona. Here we used a diblock PGA-PEG copolymer with a 57\% PEG content, whereas the corona used in the previous study consisted on a grafted PGA-PEG copolymer with a 24\% PEG content. The resulting nanocapsules were evaluated with respect to their hemocompatibility, biodistribution and blood kinetic profile. Finally, their effectiveness as carriers for anticancer drug delivery was assessed using the drug docetaxel (DCX).

\section{Materials and methods}

\subsection{Chemicals}

Docetaxel (DCX) (from Fluka), Miglyol $812^{\circledR}$, neutral oil formed by esters of caprylic and capric fatty acid and glycerol, was a gift sample from Sasol Germany GmbH (Germany). Epikuron $170^{\circledR}$, a phosphatidylcholine enriched fraction of soybean lecithin, was provided by Cargill (Spain). Benzalkonium chloride, poloxamer 188 (Pluronic $^{\circledR}$ F68) and polyglutamic acid (PAG; $M_{w} 15-50 \mathrm{kDa}$ ) were purchased from Sigma-Aldrich (Spain). Polyglutamic acidpolyethylene glycol block co-polymer (PGA-PEG; $M_{w} 35 \mathrm{kDa}$ ) was supplied by Alamanda Polymers (USA). The used PGA-PEG is a diblock copolymer with a percentage $\mathrm{w} / \mathrm{w}$ of PEG of about $57 \%$. PEG chains length was $20 \mathrm{kDa}$ and PGA chains length was about $15 \mathrm{kDa}$. The NIR (Near Infra Red) dye 1,1'-dioctadecyl-3,3,3',3'tetramethylindodicarbocyanine perchlorate (DiD) (DiD Em $644 \mathrm{~nm}$; Ex $664 \mathrm{~nm}$ ) was obtained from Molecular Probes-Invitrogen (USA). Taxotere ${ }^{\circledR}$ was provided by the Hospital Pharmacy of Angers (France).

\subsection{Preparation of PGA-PEG nanocapsules}

PGA-PEG nanocapsules were prepared following a modification of the solvent displacement technique, previously reported by our group [16]. The method involves the deposition of the coating polymer onto the oily core mainly by electrostatic interaction. Briefly, an organic phase was formed by dissolving $30 \mathrm{mg}$ of Epikuron $170^{\circledR}$ in $0.5 \mathrm{ml}$ of ethanol, followed by the addition of $125 \mu \mathrm{l}$ of Mygliol $812^{\circledR}$ and $7 \mathrm{mg}$ of the cationic surfactant, benzalkonium chloride, in $9 \mathrm{ml}$ of acetone solution. This organic phase was immediately poured over $20 \mathrm{ml}$ of an aqueous solution containing poloxamer $(0.25 \% \mathrm{w} / \mathrm{v})$ and PGA or PGA-PEG $(10 \mathrm{mg})$. Finally, solvents from the suspension were evaporated under vacuum to a final constant volume of $10 \mathrm{ml}$. PGA nanocapsules were prepared by the same method and nanoemulsions, used as controls, were also obtained by the same method with the exception that PGA was used instead of polymer or, in the case of the nanoemulsions no polymer was added. We prepared both anionic and cationic nanoemulsions which differ in the presence or not of the cationic surfactant benzalkonium chloride [15].

\subsection{Characterization of the nanostructures}

Particle size and polydispersion index of the PGA-PEG and PGA nanocapsules and uncoated nanoemulsions were determined by Dynamic Light Scattering (DLS). Samples were diluted to an appropriate concentration in deionized water and each analysis was carried out at $25^{\circ} \mathrm{C}$ with an angle detection of $173^{\circ}$. The zeta potential values were calculated from the mean electrophoretic mobility values, as determined by Laser Doppler Anemometry (LDA). For LDA measurements, samples were diluted with $\mathrm{KCl} 1 \mathrm{mM}$ and placed in an electrophoretic cell. DLS and LDA analysis were performed on three independently prepared samples using a NanoZS ${ }^{\circledR}$ (Malvern Instruments, Malvern, UK).

\subsection{DCX encapsulation in PGA-PEG nanocapsules}

DCX was incorporated to the PGA-PEG nanocapsules by adding $0.5 \mathrm{ml}$ of a DCX stock ethanol solution (conc. $20 \mathrm{mg} / \mathrm{ml}$ ) to the organic phase and then, following the process as described. PGA-PEG nanocapsules were concentrated by vacuum to a final volume of $5 \mathrm{ml}$ in order to obtain a final drug concentration of $2 \mathrm{mg} / \mathrm{ml}$.

The encapsulation efficiency of DCX in PGA-PEG nanocapsules was determined by separating the non-encapsulated drug from the DCX-loaded nanocapsules by ultracentrifugation $(27,400 \mathrm{~g}$, $15^{\circ} \mathrm{C}, 1 \mathrm{~h}$ ). The free DCX was determined in the infranatant after ultracentrifugation.

The total amount of DCX in the formulation was determined from a fresh aliquot of nanocapsules diluted in acetonitrile and centrifuged for $20 \mathrm{~min}$ at $4000 \mathrm{~g}$.

The difference between the total amount of DCX in the nanocapsules suspension and the free DCX allowed us to indirectly calculate the value of the encapsulated drug in the nanocapsules. The encapsulation efficiency was calculated as follows (Eq. (1)):

E.E. $(\%)=(A-B) / A \times 100$

where $A$ is the experimental total drug amount and $B$ is the unloaded drug amount.

DCX was analyzed by HPLC using a slightly modified version of the method proposed by Lee et al. [17]. The HPLC system consisted of an Agilent 1100 Series instrument equipped with a UV detector set at $227 \mathrm{~nm}$ and a reverse phase Zorbax Eclipse ${ }^{\circledR}$ XDB-C8 column $(4.6 \times 150 \mathrm{~mm}$ i.d., pore size $5 \mu \mathrm{m}$ Agilent USA). The mobile phase consisted of a mixture of acetonitrile and $0.1 \% \mathrm{v} / \mathrm{v}$ ortophosphoric acid $(55: 45 \mathrm{v} / \mathrm{v})$ and the flow rate was $1 \mathrm{ml} / \mathrm{min}$.

\subsection{DiD encapsulation into nanostructures}

DiD-loaded nanocapsules and DiD-loaded anionic nanoemulsion were obtained by replacing $0.5 \mathrm{ml}$ of ethanol of the organic phase with $0.5 \mathrm{ml}$ of DiD stock solution in ethanol $(2.5 \mathrm{mg} / \mathrm{ml})$. The final concentration of DiD in the nanocapsules and in the anionic nanoemulsion was around $100 \mu \mathrm{g} / \mathrm{ml}$. The efficiency of DiD encapsulation in all the systems was determined indirectly by calculating the difference between the total amount of fluorescent probe in the formulations and the free dye measured in the infranatant of the nanocapsules and nanoemulsions preparations after ultracentrifugation $\left(27,400 \mathrm{~g}, 15^{\circ} \mathrm{C}, 1 \mathrm{~h}\right)$. At the end of the 
ultracentrifugation step, aliquots of infranatant were diluted with acetonitrile and analyzed by UV spectrophotometry $(\lambda=646 \mathrm{~nm})$. To determine the total amount of probe in the different systems, fresh aliquots of nanocapsules and nanoemulsions were diluted with acetonitrile, centrifuged and analyzed at $\lambda=646 \mathrm{~nm}$.

\subsection{Hemolysis test}

The hemolytic potential of the PGA-PEG nanocapsules was determined and compared with that of the PGA nanocapsules and cationic nanoemulsion in rat blood. The blood of female Wistar rats was obtained by cardiac puncture. Sodium citrate, $\mathrm{pH} 7.4$, was diluted with blood (1:10) before adding PBS. This mixture was centrifuged $\left(700 \mathrm{~g}, 20^{\circ} \mathrm{C}, 10 \mathrm{~min}\right)$ three times; each time the supernatant was discarded and PBS added. Then, the erythrocytes were diluted with PBS $(3 \% \mathrm{w} / \mathrm{v})$ and stored at $4{ }^{\circ} \mathrm{C}$. A sample of $150 \mu \mathrm{l}$ of the erythrocytes stock dispersion was added to $150 \mu$ l of nanocapsules suspensions ( $2 \% \mathrm{w} / \mathrm{v}$ and $1 \% \mathrm{w} / \mathrm{v})$ and incubated with shaking at $37^{\circ} \mathrm{C}$ for $1 \mathrm{~h}$. In order to remove intact erythrocytes and debris, the samples were, then, centrifuged $\left(750 \mathrm{~g}, 20^{\circ} \mathrm{C}, 3 \mathrm{~min}\right)$. $100 \mu \mathrm{l}$ of the supernatant was added to $2000 \mu \mathrm{l}$ of a mixture of absolute ethanol and $\mathrm{HCl}(40 / 1(\mathrm{v} / \mathrm{v}))$ and centrifuged again. This mixture dissolved all the components and avoided hemoglobin precipitation. The absorption of the supernatant was measured by UV spectroscopy ( $\lambda=398 \mathrm{~nm}$ ) and compared against blank samples. Results were set relative to control samples with $0 \%$ lysis (PBS) and 100\% lysis (bidistilled water) [18]. The hemolytic percentage was calculated according to the equation (Eq. (2)) [19]:

Lysis $(\%)=\left(A_{\text {sample }}-A_{\text {blank }}\right) /\left(A_{\text {water }}-A_{\text {blank }}\right) \times 100$

\subsection{In vitro complement activation study}

Complement activation of PGA-PEG nanocapsules was evaluated in normal human serum (provided by the Establissment Francais du Sang, CHU, Angers, France) by measuring the residual hemolytic capacity of the complement system after coming in contact with the different particles [20]. We determined the amount of serum capable of lysing $50 \%$ of a fixed number of sensitized sheep erythrocytes with rabbit anti-sheep erythrocyte antibodies ( $\mathrm{CH} 50$ ), according to a procedure described elsewhere [21]. Complement activation was expressed as a function of the surface area. Nanocapsules and nanoemulsions surface areas were calculated as previously described [22], using the equation: $S=n 4 \pi r^{2}$ and $V=n(4 / 3)\left(\pi r^{2}\right)$ leading to $S=3 m / r \rho$ where $S$ is the surface area $\left(\mathrm{cm}^{2}\right)$ and $V$ the volume $\left(\mathrm{cm}^{3}\right)$ of $n$ spherical beads of average radius $r(\mathrm{~cm}), m$ the weight $(\mu \mathrm{g})$ and $\rho$ the volumetric mass $\left(\mu \mathrm{g} / \mathrm{cm}^{3}\right)$. All experiments were performed in triplicate [23].

\subsection{Pharmacokinetic study of DiD-loaded nanocapsules}

Animal care was provided in strict accordance with the French Ministry of Agriculture regulations. The treatment was performed according to Morille et al. [23] as follows: $150 \mu$ of DiD-loaded PGA-PEG nanocapsules was injected in the tail vein of six-week old female Swiss mice (20-22 g) (Ets Janvier, Le Genest-St-ile, France). DiD-loaded PGA nanocapsules or anionic nanoemulsion, were injected following the same procedure, and used as controls. Blood samples were withdrawn from three animals for each formulation at 30,60, 120, $240 \mathrm{~min}$ and $24 \mathrm{~h}$ after injection, transferred to BD Vacutainer tubes (Vacutainer, SST II Advance, $5 \mathrm{ml}$, Becton Dickinson France SAS, France) and centrifuged at $4000 \mathrm{rpm}$ for $10 \mathrm{~min}$. $150 \mu \mathrm{l}$ of the supernatant was deposited in a black, 96-well plate (Greiner Bio-one, Germany) and its optical density measured using a Fluoroskan (Fluoroskan 262 Ascent, FL,
USA). To obtain the fluorescence at time 0 , aliquots of fluorescent nanocapsules were diluted with a blood sample at the same in vivo concentration. Plasma residual fluorescence was measured from the supernatant of centrifuged blood taken from three rats receiving $150 \mu \mathrm{l}$ of a physiological saline solution.

DiD fluorescence was measured by using a Fluoroscan at excitation wavelength of $644 \mathrm{~nm}$ with an emission wavelength of $664 \mathrm{~nm}$. The blood concentration of the different systems at the various times was calculated on the assumption that blood represents $7.5 \%$ of mouse body weight [24]. Fluorescence was expressed in fluorescence units (FU) and was calculated as: FU sample (treated) - FU empty (control serum); $100 \%$ of fluorescence was considered as the value at $t=0 \mathrm{~min}$ [23].

Pharmacokinetic data were treated by a non-compartmental analysis of the percentage of the injected dose versus time profiles with Kinetica 5.1 software (Thermo Fischer Scientific, France). The elimination half-live was calculated as follows (Eq. (3)):

$t_{1 / 2}=\log (2) / \mathrm{Lz}$

where $L z$ was determined from linear regression using the data corresponding to the terminal phase. The trapezoidal rule was used to calculate the area under the curve (AUC) during the whole experimental period (AUC [0-24h]) without extrapolation, as well as the area under the first moment curve (AUMC). The mean residence time was calculated from 0 to $24 \mathrm{~h}$, from the following equation (Eq. (4)):

MRT $[0-24 \mathrm{~h}]=$ AUMC $[0-24 \mathrm{~h}] /$ AUC $[0-24 \mathrm{~h}]$

\subsection{In vivo fluorescence imaging}

The non-invasive biofluorescence imaging (BFI) of PGA, PGAPEG nanocapules and nanoemulsions was performed in healthy nude mice at $1 \mathrm{~h}, 3 \mathrm{~h}, 5 \mathrm{~h}, 24 \mathrm{~h}$ and $48 \mathrm{~h}$ post-injection, using DiD as near-infrared (NIR) fluorophore. Besides, to avoid hair auto-fluorescence, the animals were fed for 2 weeks without chlorophyll. The fluorescence imaging system was a NightOWL II (Berthold Technologies, Germany) equipped with cooled, slow-scan CCD camera and driven with the WinLight 32 software (Berthold Technology, Germany). With the BFI system, the fluorescent acquisition time was of $3 \mathrm{~s}$ and the fluorescent signal was overlaid on a picture of each mouse.

According to the fluorescent characteristics of the DiD fluorescent tag used to localize the nanosystems, the $590 \mathrm{~nm}$ excitation and $655 \mathrm{~nm}$ emission filters were selected. In parallel, the light beam was kept constant for each fluorescent measurement, which was ideal with the ringlight, epi-illumination. As the ringlight was always set at the same height, the excitation energy on the sample would always be the same.

Each mouse was anesthesized with a $4 \%$ air-isofluran blend. Once placed in the acquisition chamber, the anesthesia of the mice was maintained with a $2 \%$ air-isofluran mixture throughout the experiment as described above [25].

\subsection{In vivo antitumor efficacy study}

\subsubsection{Tumor cell line}

U87MG glioma cell line (ATCC, Manassas, VA) was obtained from the European Collection of Cell Culture (UK, $\mathrm{N}^{\circ} 94110705$ ). The cells were cultured at $37{ }^{\circ} \mathrm{C} / 5 \% \mathrm{CO}_{2}$ in Dulbecco modified eagle medium (DMEM) with glucose and L-glutamine (BioWhitakker, Belgium) containing $10 \%$ fetal calf serum (FCS) (BioWhitakker) and $1 \%$ antibiotic and antimycotic solution (Sigma, France). On the implantation day, cells were trypsinized and resuspended in minimal essential medium (EMEM), without FCS or antibiotics, to the final desired concentration [24]. 


\subsubsection{Subcutaneous glioma model and therapy schedule}

Animals were manipulated under isoflurane/oxygen anesthesia. Tumor bearing mice were prepared by subcutaneous injection of a suspension of $1 \times 10^{6}$ U87MG glioma cells in $150 \mu \mathrm{l}$ of Hanks Balanced Saline Solution (HBSS) into the right flank of athymic nude mice (6 weeks old females, 20-24 g, purchased from Ets Janvier, Le Genest-St-ile, France). Tumor growth was tracked by regularly measuring the length and width of tumors with a caliper. The tumor volume $(V)$ was estimated by the mathematical ellipsoid formula (Eq. (5)):

$V=(\Pi / 6) \times(\text { width })^{2} \times($ length $)$

When tumors reached a calculated average volume of approximately $200 \mathrm{~mm}^{3}$, the mice were randomized into three groups to ensure that the initial tumor volumes on the day of treatment were not significantly different among groups. Animals were treated (Day 0 ) by a single IV injection of $150 \mu$ l of the different treatments, via lateral tail vein as follows: physiological saline solution $(0.9 \% \mathrm{NaCl})$, DCX-loaded PGA-PEG nanocapsules (20 mg/kg mouse) and Taxotere $^{\circledR}$ (20 mg/kg mouse).

Tumor size was measured twice weekly after the intravenous administration of the treatments. At day 21, mice were then isolated and weighed. The treated groups were compared in terms of mean survival time in days after U87GM cell implantation. The percentage of the increase in survival time (\%IST) was determined relative to the mean survival of untreated controls as presented in the following equation (Eq. (6)):

$\%$ IST $=\operatorname{Mean}_{T}-$ Mean $_{C} /$ Mean $_{C} \times 100$

where Mean $\mathrm{T}_{\mathrm{T}}$ was the mean of survival time of the treated group and Mean $_{C}$ was the median/mean of the survival time of the control group [26].

\subsection{Statistical analysis}

Statistical analysis of the pharmacokinetic data was conducted using the non-parametric Kruskal-Wallis method followed by the Tukey HSD multiple comparison test $(p<0.05$ was considered as significant).

For the estimation of the mean survival times, we used a censored model, the Kaplan Meier plot, according to which censored events were both tumor growth and the end of the evaluation period, assuming that in both cases deaths occur before the next size control of the tumor. More specifically, tumor burden that exceed $10 \%$ of the animal's normal weight was considered as sacrificed parameter according to regulatory ordinances. Statistical significance was calculated using the log-rank test (Mantel-Cox test); SPSS software version 16.0 (SPSS Inc.) was used for that purpose. The different treatment groups were compared in terms of range, and mean survival time (days), long term survivors (\%) and increase in survival time (IST mean ).

\section{Results and discussion}

Recently we reported the development of nanocapsules made of a PGA-PEG grafted copolymer, with a PEG content of $24 \% \mathrm{w} / \mathrm{w}$ and their use for the delivery of the anticancer drug plitidepsin [15]. Here, we report an updated version of this nanocarrier containing a highly PEGylated di-block PGA-PEG copolymer (PEG content 57\% $\mathrm{w} / \mathrm{w}$ ) and we compared its behavior with the behavior of the nonPEGylated PGA nanocapsules. The rationale behind the use of this polymer was to assess the role of the polymer shell of the nanocapsules on their toxicity, biodistribution and capacity to modify the efficacy of the anticancer drug DCX. Hence, in the following paragraphs we present: (i) the development and characterization of a new type of PGA-PEG nanocapsules; (ii) their hemolysis and complement activation activity; (iii) their biodistribution and blood kinetics profile; (iv) their efficacy when loaded with DCX in controlling tumor growth and increasing the survival rate of a U87MG glioma mice model, using the commercial formulation Taxotere ${ }^{\circledR}$ as a control.

\subsection{Characteristics of the PGA-PEG nanocapsules and control formulations}

PGA-PEG nanocapsules were prepared using the solvent displacement technique previously reported [15]. The nanocapsules were loaded separately with DiD and DCX and, then, characterized by their size, zeta potential and encapsulation efficiency. Table 1 shows the characteristics of this novel formulation, and also those of PGA nanocapsules and the uncoated nanoemulsions used as controls. Regardless of whether they had a loaded compound or a polymer coating, the nanocapsules formed monodispersed populations with a mean size of around $200 \mathrm{~nm}$. As expected, PGA-PEG nanocapsules were negatively charged, although their zeta potential values were lower than those of PGA nanocapsules $(-20 \mathrm{mV}$ versus $-39 \mathrm{mV}$ for blank nanosystems). This was attributed to the shielding effect of large PEG chains located around the surface of the systems. Table 1 also shows the charge inversion observed for PGA-PEG and PGA nanocapsules with respect to their oily cores $(+40 \mathrm{mV})$, thus evidencing the formation of a continuous shell of polymer around the cationic oily nanodroplets. The anionic nanoemulsion exhibited a negative potential $(-40 \mathrm{mV})$, which was attributed to the use of lecithin for their stabilization.

As indicated in Section 1, these nanocapsules were designed to have a high capacity to load hydrophobic drugs. As expected, the hydrophobic drug DCX could be efficiently encapsulated (90\% encapsulation efficiency). On the other hand, the fluorescent marker, DiD, could also be loaded, but due to its amphiphilic character, its encapsulation efficiency was slightly reduced. As indicated in Table 1, the original characteristics of nanocapsules were not altered upon encapsulation of either the drug or the fluorescent marker.

An additional critical property of anticancer-loaded nanocarriers is their stability upon dilution in physiological media. Interestingly, the results of this study showed that upon dilution with PBS at $37^{\circ} \mathrm{C}$, PGA-PEG nanocapsules were stable during at least $24 \mathrm{~h}$.

\subsection{Hemolytic activity of the PGA-PEG nanocapsules}

This analysis was performed in order to have an estimate of the nanocapsules hemocompatibility after IV administration. The release of hemoglobin was used to quantify the membrane damage caused for the systems assayed. The results showed that the PGA-PEG nanocapsules had no hemolytic behavior when diluted

Table 1

Physicochemical characteristics of blank, DiD- and DXC-loaded PGA-PEG nanocapsules. The characteristics of PGA nanocapsules, and cationic and anionic nanoemulsions, used as controls are also shown. P.I.: polydispersity index; E.E.: Encapsulation efficiency. Values are given as mean \pm SD $(n=3)$. NCs: nanocapsules and NE: nanoemulsions.

\begin{tabular}{lllll}
\hline Formulation & Size $(\mathrm{nm})$ & P.I. & $\zeta$ Potential $(\mathrm{mV})$ & E.E. $(\%)$ \\
\hline Blank PGA-PEG NCs & $180 \pm 4$ & 0.1 & $-20 \pm 4$ & - \\
Blank PGA NCs & $183 \pm 6$ & 0.1 & $-39 \pm 4$ & - \\
Blank anionic NE & $207 \pm 7$ & 0.1 & $-38 \pm 1$ & - \\
Blank cationic NE & $227 \pm 8$ & 0.1 & $+40 \pm 4$ & - \\
DiD-loaded PGA-PEG NCs & $194 \pm 2$ & 0.1 & $-15 \pm 3$ & $70 \pm 8$ \\
DiD-loaded PGA NCs & $179 \pm 3$ & 0.1 & $-31 \pm 2$ & $67 \pm 5$ \\
DiD-loaded anionic NE & $214 \pm 5$ & 0.1 & $-28 \pm 6$ & $79 \pm 10$ \\
DCX-loaded PGA-PEG NCs & $200 \pm 3$ & 0.1 & $-20 \pm 4$ & $90 \pm 2$ \\
\hline
\end{tabular}


with erythrocytes at $1 \%$ and $0.5 \% \mathrm{w} / \mathrm{v}$, being the hemolysis values under $3 \%$. Similar values were obtained for the PGA nanocapsules with no detectable disturbance in the red blood cell membranes. In contrast, the cationic nanoemulsion, used as a control, had a high hemolytic activity (60\%) at the concentrations tested. Therefore, these results indicate that the presence of a PGA-PEG or PGA shell around the oily nanodroplets counteracts the inherent hemolytic activity of a cationic nanoemulsion, thus providing the system with an adequate hemocompatibility.

\subsection{Complement activation properties of PGA-PEG nanocapsules}

It is known that following injection into the blood circulation, foreign particles can be rapidly cleared by MPS phagocytosis. This process involves first, the recognition of these particles by opsonins, complement proteins which upon adsorption onto the nanoparticles facilitate their recognition and elimination by MPS. In this study, the complement activation properties of the nanocapsules were evaluated in order to gain some insights on the in vivo fate of nanocarriers after IV administration. With this objective in mind, we determined the extent of the interactions between the nanocarriers and the complement system. Using the $\mathrm{CH} 50$ technique, we measured the hemolytic capacity of a fixed amount of normal human serum against 50\% of antibody-sensitized sheep erythrocytes previously exposed to different concentrations of the PGA-PEG nanocapsules. PGA nanocapsules and the cationic nanoemulsion were used as controls.

As presented in Fig. 1, PGA-PEG and PGA nanocapsules exhibited a very low dose-dependent complement activation capacity, whereas the cationic emulsion used as control triggered a rapid complement activation. These results are in accordance with those previously reported in the literature, which indicate that positively charged particles are prone to lead to complement activation activity [27], whereas the use of anionic PGA-PEG counteracts this activity [28,29].

\subsection{In vivo studies}

\subsubsection{Blood kinetics of PGA-PEG nanocapsules}

Fluorescently-labeled PGA-PEG nanocapsules and the control formulations, PGA nanocapsules and anionic nanoemulsion, were intravenously injected in mice to study their kinetics in blood. The results indicated that the nanoemulsion was removed from

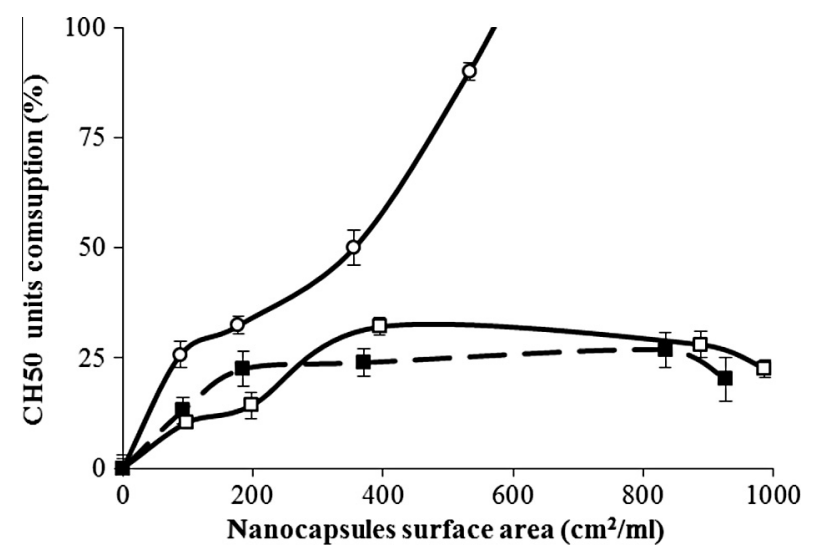

Fig. 1. Complement activation profile measured as lytic capacity of the serum (\% CH50 units) toward antibody-sensitized sheep erythrocytes after exposure to PGA-PEG nanocapsules ( $\square$ ) compared to PGA nanocapsules ( $\square$ ) and the cationic nanoemulsion $(\bigcirc)$ used as controls. Complement consumption was evaluated as a function of the nanoparticles surface area $\left(\mathrm{cm}^{2}\right)$. the blood circulation within a few minutes, with only $15 \%$ of the total fluorescence remaining in plasma $30 \mathrm{~min}$ after IV injection (Fig. 2). In contrast, the PGA-PEG nanocapsules concentration remained high (about $40 \%$ of injected dose) 3 h post-injection. This concentration decreased slowly overtime, to a value of $20 \%$ of the injected dose after $24 \mathrm{~h}$. This value was slightly lower for PGA nanocapsules ( $8 \%$ of the injected dose remained in the circulation at $24 \mathrm{~h}$ ). The pharmacokinetic parameters in plasma are summarized in Table 2. It can be noted that the behavior of both, PGA-PEG and PGA nanocapsules, significantly differs from that of the control nanoemulsion (Kruskal-Wallis and Tukey HSD multiple comparison test, $p<0.05$ ). Particularly remarkable is the difference in the elimination half-life and mean residence time (MRT), whose values in the case of PEG-PGA nanocapsules are double than those observed for the control nanoemulsion. Moreover, the values of the area under the curve (AUC) rose from $11 \mathrm{mg} / \mathrm{ml} \mathrm{h}$ for the nanoemulsion to $50 \mathrm{mg} / \mathrm{ml} \mathrm{h}$ for the PGA-PEG nanocapsules. Finally, it was also observed that a high PEGylation of the polymer shell of the nanocapsules led to a significant increase in their elimination half-life, MRT and AUC values.

Overall, these results illustrate the protective role the PGA-PEG and PGA coatings have vis a vis the phagocytic uptake of the nanocarrier by the MPS. Moreover, in agreement with previous reports, our results indicate that, by modifying the PGA with PEG, it is possible to further enhance this protective role [30,31]. This process may be explained by the formation of a more protective shield,

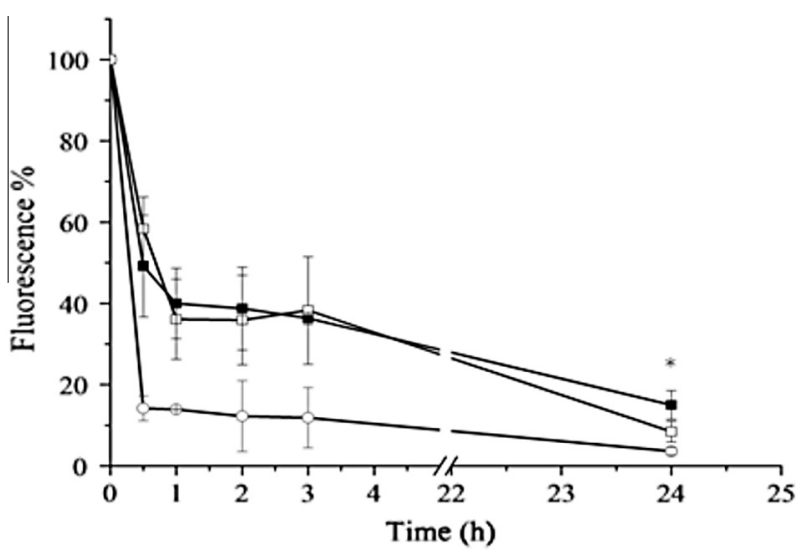

Fig. 2. Percentage of nanocarriers-associated fluorescence remaining in plasma after single bolus inyection in mice of PGA-PEG nanocapsules $(\boldsymbol{\square})$. The fluorescence observed upon administration of PGA nanocapsules $(\square)$ and the uncoated nanoemulsion $(\bigcirc)$ are also showed as controls. The injected dose of DiD was $1 \mathrm{mg} / \mathrm{kg}$. Each data point represents the group mean \pm SD of the percentage of injected dose. *Significant differences with respect to the control nanoemulsion; $p<0.05$, KruskalWallis analysis, followed by the Tukey HSD multiple comparison test.

Table 2

Main parameters illustrating the plasma pharmacokinetics of DiD-loaded PGA-PEG nanocapsules after a single IV injection in Swiss mice. Pharmacokinetic parameters of DiD-loaded PGA nanocapsules and uncoated anionic nanoemulsion are also shown. Each data point represents the group mean \pm SD. NCs: nanocapsules and NE: nanoemulsions.

\begin{tabular}{lccl}
\hline Formulation & $t^{1 / 2}$ Elimination $(\mathrm{h})$ & MRT $(\mathrm{h})$ & AUC $(\mathrm{mg} / \mathrm{ml} \mathrm{h})$ \\
\hline PGA-PEG NCs & $16.08 \pm 0.9^{* *}$ & $17.00 \pm 3.6^{* *}$ & $50.65 \pm 5.7^{* *}$ \\
PGA NCs & $10.02 \pm 0.83^{*}$ & $9.72 \pm 1.7^{*}$ & $38.02 \pm 1.4^{*}$ \\
NE & $8.17 \pm 1.0$ & $6.59 \pm 2.0$ & $11.43 \pm 2.2$ \\
\hline
\end{tabular}

Significant differences with respect to the NE.

* Significant different with respect to the NE and PGA nanocapsules $(p<0.05)$; Kruskal-Wallis analysis, followed by the Tukey HSD multiple comparison test. 


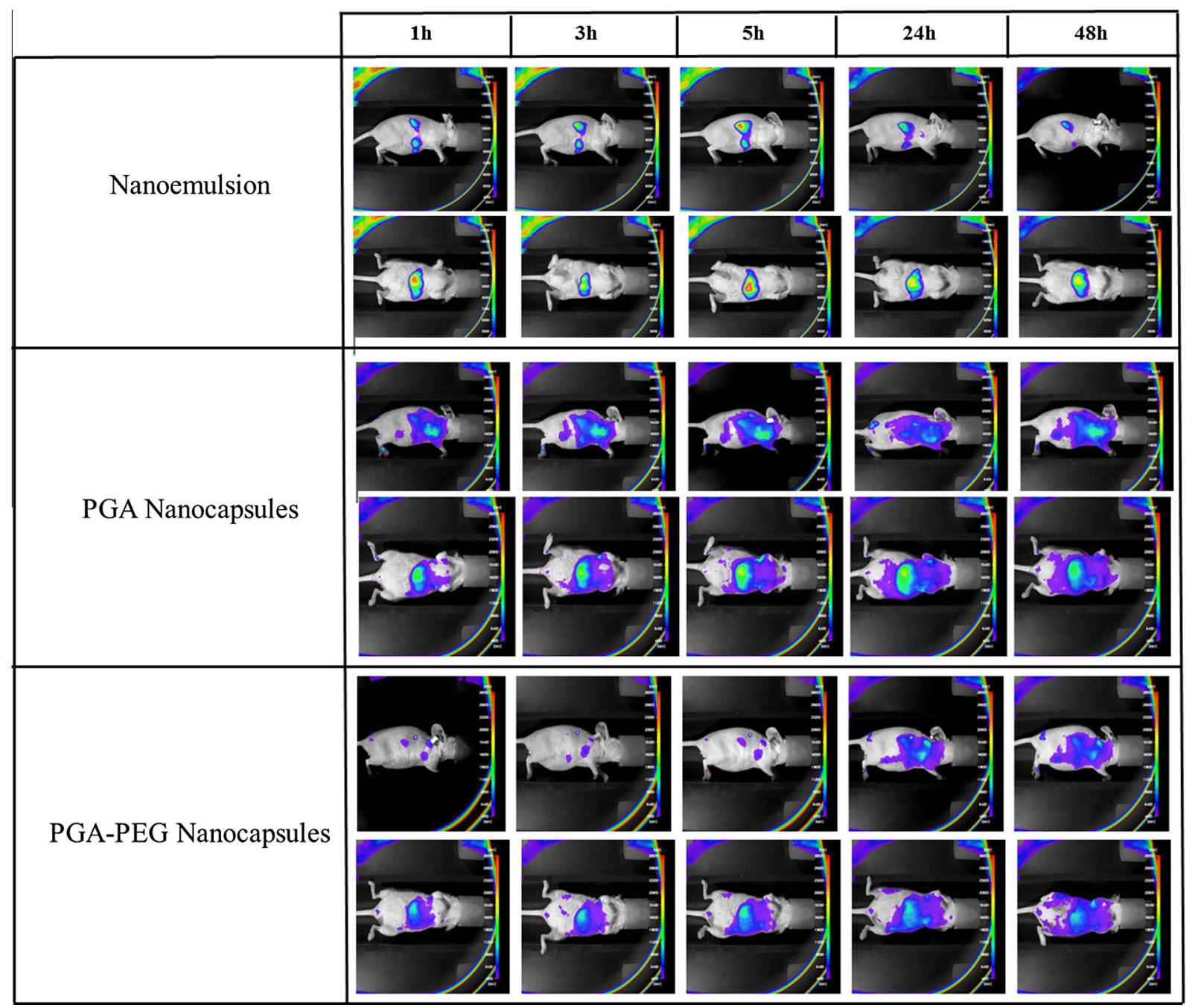

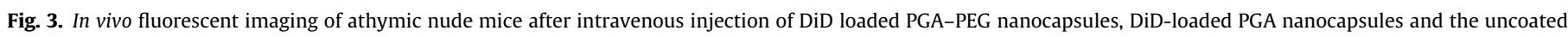

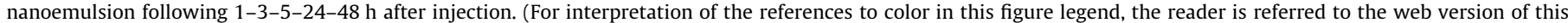
article.)

which through steric repulsion to opsonins is able to prevent the rapid elimination of the nanoparticles from the blood circulation.

\subsubsection{Biodistribution of PGA-PEG nanocapsules using in vivo fluorescence imaging}

After the DiD-labeled systems were injected intravenously in the tail vein of mice, we followed the distribution of the PGAPEG nanocapsules using a NIR biofluorescence imaging system. PGA nanocapsules and the anionic nanoemulsion were used as controls. The DiD is a NIR label frequently used to follow the biodistribution of lipid carriers. Images were taken at $1 \mathrm{~h}, 3 \mathrm{~h}, 5 \mathrm{~h}$, $24 \mathrm{~h}$, and $48 \mathrm{~h}$, from lateral and decubitus dorsal views to obtain a general overview of the nanocapsules biodistribution, and identify the organs and subjacent tissues affected. As shown in Fig. 3, the nanoemulsion was rapidly eliminated from the blood and mostly localized in the liver, where the fluorescence signal was very intense. A different biodistribution was observed for PGA and PGA-PEG nanocapsules. In these cases, the NIR signal was found distributed in the whole body of the animal and a significant amount of fluorescence remained in circulation up to $48 \mathrm{~h}$. These results are in agreement with those of the blood elimination kinetics; although in this qualitative analysis no difference in the biodistribution patterns could be observed for PGA and PGA-PEG nanocapsules.

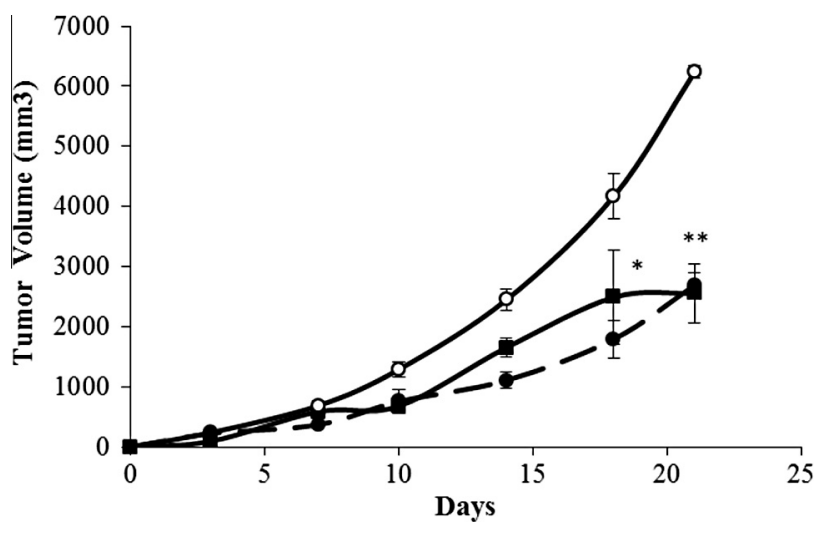

Fig. 4. Evolution of tumor volume following IV administration of a single-dose of DCX-loaded PGA-PEG nanocapsules $(\boldsymbol{\square})$ and Taxotere $^{\circledR}(\mathbf{0})$ in a subcutaneous U87MG glioma mouse model. Control group received $0.9 \% \mathrm{NaCl}$ solution $(\bigcirc)$. Statistical analysis by pairs shows significant differences on Day 18 and 21 in tumor growth of mice treated with PGA-PEG nanocapsules or Taxotere ${ }^{\circledR}$ as compared to control (non-treated mice); ${ }^{*} p<0.05,{ }^{* *} p<0.01$, Kruskal-Wallis analysis, followed by the Tukey HSD multiple comparison test. All data are reported as means \pm SD; $n=6$. 
Table 3

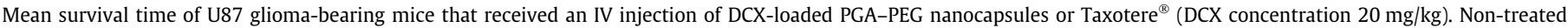
mice (receiving an injection of serum) were used as a control. Number of mice per group: 6 .

\begin{tabular}{|c|c|c|c|c|}
\hline \multirow[t]{2}{*}{ Treatment } & \multicolumn{2}{|c|}{ Mean survival time (days) } & \multicolumn{2}{|c|}{ Increase in survival time (IST) (\%) } \\
\hline & Survival time range & Mean \pm SD & IST $_{\text {mean }}$ & $p$-Value versus control \\
\hline DCX-loaded PGA-PEG NCs & $7-18$ & $18.1 \pm 2$ & 61.6 & 0.066 \\
\hline Taxotere $^{\circledR}$ & $7-21$ & $16.8 \pm 2$ & 50 & 0.147 \\
\hline Control (non-treated) & $7-17$ & $11.2 \pm 2$ & - & - \\
\hline
\end{tabular}

\%IST percentage of increase in survival time relative to that of the serum control.

Overall, our blood kinetics and biodistribution profiles results indicate that both PGA and PGA-PEG have a prolonged blood circulation and are widely distributed in the body.

\subsubsection{DCX-loaded PGA-PEG nanocapsules in vivo antitumor activity}

The promising pharmacokinetics results obtained with PGAPEG nanocapsules encouraged us to evaluate their efficacy in a U87MG glioma bearing mice model. For this study we used the commercial DCX formulation, Taxotere ${ }^{\circledR}$, as a control. The results presented in Fig. 4 show the growth of the subcutaneous tumor over time and the tumor volume at days 18 and 21 as compared to the initial volume (at the day of the treatment). The results show that in the control group, the tumor grew exponentially, reaching a volume of about $6 \times 10^{3} \mathrm{~mm}^{3}$ at day 20 . In contrast, upon intravenous injection of a single dose of either, DCX-loaded PGA-PEG nanocapsules or Taxotere ${ }^{\circledR}$, a significant decrease in the tumor growth $(p<0.01)$ was observed. Interestingly, toward the end of the study a tendency to reach a plateau in the tumor size was noted for the mice tumors treated with nanocapsules, whereas the size of the tumors of mice treated with Taxotere ${ }^{\circledR}$ continued to increase.

\subsection{Survival study}

As an additional relevant study, we compared the survival rate of mice treated with PGA-PEG nanocapsules to those treated with

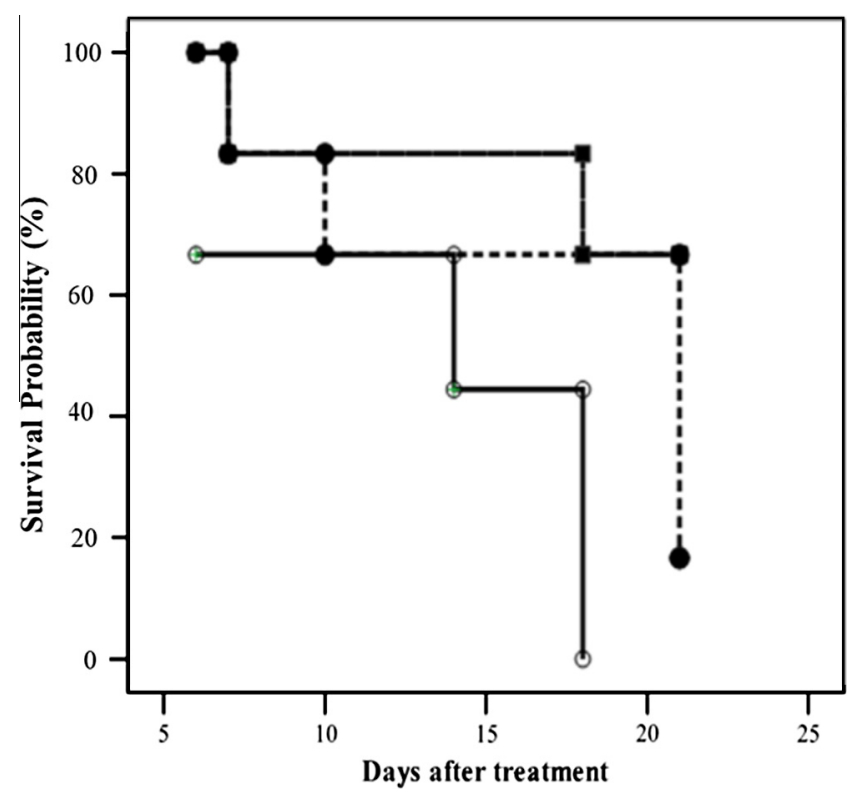

Fig. 5. Kaplan-Meier survival curves of subcutaneous U87MG glioma tumor bearing mice following treatment with Taxotere ${ }^{\circledR}(\bullet)$ or DCX-loaded PGA-PEG nanocapsules $(\boldsymbol{\square})$. Non-treated mice were used as a control $(O)$. (For interpretation of the references to color in this figure legend, the reader is referred to the web version of this article.)
Taxotere ${ }^{\circledR}$. Descriptive and statistical data from the survival study are presented in Table 3. Survival curves according to the KaplanMeier method are showed in Fig. 5. Our results indicate that nontreated mice did not survive beyond 18 days, whereas the survival time increased significantly upon treatment with Taxotere ${ }^{\circledR}$ and, even more so, upon treatment with DCX-loaded PGA-PEG nanocapsules. More precisely, the results in Table 3 show an increase in the mean survival time of mice treated with commercial Taxotere ${ }^{\circledR}$ of $50 \%$ over the control, versus a $62 \%$ increase in those treated with the PGA-PEG nanocapsules. These results clearly show a beneficial effect in the survival rate of the animals after treatment with DCX-loaded PGA-PEG nanocapsules. Overall, the advantage of using PGA-PEG nanocapsules instead of Taxotere $^{\circledR}$ relies on the lack of toxic surfactants in the former formulation. Surfactants such as sorbitan monooleate are known to significantly contribute to the toxicity of Taxotere ${ }^{\circledR}$. Furthermore, we also suggest that a more adequate biodistribution of the drug occurs in mice treated with PGA-PEG nanocapsules, which could eventually lead to an increase in the passive accumulation of loaded nanocapsules in the tumor target site.

\section{Conclusion}

We report here the first evidence found in vivo supporting PGAPEG nanocapsules as potential candidates to act as carriers of the anticancer drug DCX. Specifically, our results show that these nanocarriers have good blood compatibility and a very low complement activity. They also show that the hydrophilic shell (either PGA or PGA-PEG) allows the nanocapsules to remain longer in the blood stream, and that this effect is particularly remarkable for the PEGylated PGA nanocapsules. Finally, DCX-loaded PGA-PEG nanocapsules exhibited an antitumor effect that was comparable to that of the commercial formulation Taxotere ${ }^{\circledR}$, while they simultaneously led to significant increase in the mice survival time. Consequently, we propose the PGA-PEG nanocapsules as a new delivery technology for hydrophobic anticancer drugs.

\section{Acknowledgements}

This work was supported by the European Commission FP7 EraNet - EuroNanoMed Program - Instituto de Salud Carlos III (Lymphotarg proyect, Ref. PS09/02670) and the Xunta de Galicia (Competitive Reference Groups-FEDER funds Ref. GRC2014/043. Giovanna Lollo acknowledges a fellowship from the Spanish Ministry of Education.

\section{References}

[1] M.L. Etheridge, S.A. Campbell, A.G. Erdman, C.L. Haynes, S.M. Wolf, J. McCullough, The big picture on nanomedicine: the state of investigational and approved nanomedicine products, Nanomed.: Nanotechnol. Biol. Med. 9 (2013) 1-14.

[2] O. Nag, V. Awasthi, Surface engineering of liposomes for stealth behavior, Pharmaceutics 5 (2013) 542-569.

[3] R. Gref, M. Lück, P. Quellec, M. Marchand, E. Dellacherie, S. Harnisch, T. Blunk, R.H. Müller, 'Stealth' corona-core nanoparticles surface modified by 
polyethylene glycol (PEG): influences of the corona (PEG chain length and surface density) and of the core composition on phagocytic uptake and plasma protein adsorption, Colloids Surf., B 18 (2000) 301-313.

[4] C. Oerlemans, W. Bult, M. Bos, G. Storm, J. Nijsen, W. Hennink, Polymeric micelles in anticancer therapy: targeting, imaging and triggered release, Pharmaceut. Res. 27 (2010) 2569-2589.

[5] T. Vladimir, Tumor delivery of macromolecular drugs based on the EPR effect, Adv. Drug Deliv. Rev. 63 (2011) 131-135.

[6] B. Romberg, J.M. Metselaar, L. Baranyi, C.J. Snel, R. Bünger, W.E. Hennink, J. Szebeni, G. Storm, Poly(amino acid)s: promising enzymatically degradable stealth coatings for liposomes, Int. J. Pharm. 331 (2007) 186-189.

[7] J.M. Metselaar, P. Bruin, L.W.T. de Boer, T. de Vringer, C. Snel, C. Oussoren, M.H.M. Wauben, D.J.A. Crommelin, G. Storm, W.E. Hennink, A novel family of Lamino acid-based biodegradable polymer-lipid conjugates for the development of long-circulating liposomes with effective drug-targeting capacity, Bioconjug. Chem. 14 (2003) 1156-1164.

[8] L.S. Nair, C.T. Laurencin, Biodegradable polymers as biomaterials, Prog. Polym. Sci. 32 (2007) 762-798.

[9] J.V. González-Aramundiz, M.V. Lozano, A. Sousa-Herves, E. Fernandez-Megia, N. Csaba, Polypeptides and polyaminoacids in drug delivery, Exp. Opin. Drug Deliv. 9 (2012) 183-201.

[10] L. Chun, Poly(L-glutamic acid)-anticancer drug conjugates, Adv. Drug Deliv. Rev. 54 (2002) 695-713.

[11] W. Song, M. Li, Z. Tang, Q. Li, Y. Yang, H. Liu, T. Duan, H. Hong, X. Chen, Methoxypoly(ethylene glycol)-block-poly(L-glutamic acid)-loaded cisplatin and a combination with iRGD for the treatment of non-small-cell lung cancers, Macromol. Biosci. 12 (2012) 1514-1523.

[12] J.W. Singer, Paclitaxel poliglumex (XYOTAX'TM, CT-2103): a macromolecular taxane, J. Control. Release 109 (2005) 120-126.

[13] R. Plummer, R.H. Wilson, H. Calvert, A.V. Boddy, M. Griffin, J. Sludden, M.J. Tilby, M. Eatock, D.G. Pearson, C.J. Ottley, Y. Matsumura, K. Kataoka, T. Nishiya, A phase I clinical study of cisplatin-incorporated polymeric micelles (NC-6004) in patients with solid tumours, Br. J. Cancer 104 (2011) 593-598.

[14] Y. Bae, K. Kataoka, Intelligent polymeric micelles from functional poly(ethylene glycol)-poly(amino acid) block copolymers, Adv. Drug Deliv. Rev. 61 (2009) 768-784.

[15] T. Gonzalo, G. Lollo, M. Garcia-Fuentes, D. Torres, J. Correa, R. Riguera, E. Fernandez-Megia, P. Calvo, P. Avilés, M.J. Guillén, M.J. Alonso, A new potential nano-oncological therapy based on polyamino acid nanocapsules, J. Control. Release 169 (2013) 10-16.

[16] P. Calvo, C. Remuñán-López, J. Vila-Jato, M. Alonso, Development of positively charged colloidal drug carriers: chitosan-coated polyester nanocapsules and submicron-emulsions, Colloid Polym. Sci. 275 (1997) 46-53.

[17] S.H. Lee, S.D. Yoo, K.H. Lee, Rapid and sensitive determination of paclitaxel in mouse plasma by high-performance liquid chromatography, J. Chromatogr. B 724 (1999) 357-363.
[18] M.A. Schubert, C.C. Müller-Goymann, Characterisation of surface-modified solid lipid nanoparticles (SLN): influence of lecithin and nonionic emulsifier, Eur. J. Pharm. Biopharm. 61 (2005) 77-86.

[19] J. Lu, S.C. Owen, M.S. Shoichet, Stability of self-assembled polymeric micelles in serum, Macromolecules 44 (2011) 6002-6008.

[20] M.D. Kazatchkine, M.P. Carreno, Activation of the complement system at the interface between blood and artificial surfaces, Biomaterials 9 (1988) 30-35.

[21] A. Vonarbourg, C. Passirani, P. Saulnier, P. Simard, J.C. Leroux, J.P. Benoit Evaluation of pegylated lipid nanocapsules versus complement system activation and macrophage uptake, J. Biomed. Mater. Res., Part A 78A (2006) 620-628.

[22] C. Passirani, G. Barratt, J.-P. Devissaguet, D. Labarre, Interactions of nanoparticles bearing heparin or dextran covalently bound to poly(methyl methacrylate) with the complement system, Life Sci. 62 (1998) 775-785.

[23] M. Morille, T. Montier, P. Legras, N. Carmoy, P. Brodin, B. Pitard, J.-P. Benoît, C. Passirani, Long-circulating DNA lipid nanocapsules as new vector for passive tumor targeting, Biomaterials 31 (2010) 321-329.

[24] N. Huynh, M. Morille, J. Bejaud, P. Legras, A. Vessieres, G. Jaouen, J.-P. Benoit, C. Passirani, Treatment of 9L gliosarcoma in rats by ferrociphenol-loaded lipid nanocapsules based on a passive targeting strategy via the EPR effect, Pharm. Res. 28 (2011) 3189-3198.

[25] S. David, C. Passirani, N. Carmoy, M. Morille, M. Mevel, B. Chatin, J.-P. Benoit, T Montier, B. Pitard, DNA nanocarriers for systemic administration: characterization and in vivo bioimaging in healthy mice, Mol. Ther. Nucleic Acid 2 (2013) e64.

[26] E. Allard, N.T. Huynh, A. Vessieres, P. Pigeon, G. Jaouen, J.P. Benoit, C. Passirani, Dose effect activity of ferrocifen-loaded lipid nanocapsules on a 9L-glioma model, Int. J. Pharm. 379 (2009) 317-323.

[27] A. Vonarbourg, C. Passirani, P. Saulnier, J.-P. Benoit, Parameters influencing the stealthiness of colloidal drug delivery systems, Biomaterials 27 (2006) 43564373.

[28] B.C. Dash, G. Réthoré, M. Monaghan, K. Fitzgerald, W. Gallagher, A. Pandit, The influence of size and charge of chitosan/polyglutamic acid hollow spheres on cellular internalization, viability and blood compatibility, Biomaterials 31 (2010) 8188-8197.

[29] S.M. Moghimi, A.J. Andersen, D. Ahmadvand, P.P. Wibroe, T.L. Andresen, A.C Hunter, Material properties in complement activation, Adv. Drug Deliv. Rev. 63 (2011) 1000-1007.

[30] M. Yasuhiro, Poly (amino acid) micelle nanocarriers in preclinical and clinical studies, Adv. Drug Deliv. Rev. 60 (2008) 899-914.

[31] V.C.F. Mosqueira, P. Legrand, J.-L. Morgat, M. Vert, E. Mysiakine, R. Gref, J.-P. Devissaguet, G. Barratt, Biodistribution of long-circulating PEG-grafted nanocapsules in mice: effects of PEG chain length and density, Pharm. Res. 18 (2001) 1411-1419. 